\title{
The auditory tau and kappa effects for speech and nonspeech stimuli
}

\author{
SUMI SHIGENO \\ Kitasato University, Kanagawa, Japan
}

\begin{abstract}
The tau effect, an effect of temporal intervals on the perception of spatial separation or sensory difference, has been observed in various sense modalities, including pitch bisection judgments in audition. However, systematic studies on the mechanism of the auditory tau effect have been rather scarce. In the present study, experiments were carried out using the $A X B$ method to investigate the auditory tau effect on the pitch perception of pure tones (Experiment 1 ), the auditory tau effect on perception of the $/ \mathrm{e} /-\mathrm{i} /, / \mathrm{i} / / \mathrm{e} /, / \mathrm{/} /-\mathrm{i} /$, and $/ \mathrm{i} /-\mathrm{I} /$ continua (Experiment 2 ), and the kappa effect-an effect of sensory differences on the perception of temporal intervals-on the perception of pure tones and synthetic vowels (Experiment 3 ). The results demonstrated that the auditory tau effect occurs not only in the pitch perception of pure tones, but also in the phonetic perception of the $/ \mathrm{I} / / \mathrm{i} /$ and $/ \mathrm{i} /-\mathrm{/} / \mathrm{c}$ continua of synthetic vowels. The reverse pattern of results was obtained in the /e/-/i/ and / $/$ //e/ continua. The kappa effect was found both in the pitch perception of pure tones and in phonetic judgments of synthetic vowels. These findings suggest that the interaction between temporal interval and pitch (or phonetic) perception can be explained on the basis of the integration of forward and backward context effects, and that the auditory tau and kappa effects occur at an early stage of auditory information processing.
\end{abstract}

Many experiments have been conducted on the timespace relationship in perception. For example, the tau effect is usually cited as evidence for the dependence of space perception on time. The tau effect was first identified by Helson (1930) in tactile perception. He found that when two points are stimulated successively, the perceived distance between the points varies as a function of the temporal interval separating the two points. More specifically, if three points are marked off on an observer's skin and the temporal interval between the stimulation of the first and second points is greater than that between the second and third, the observer reports that the spatial distance between the first and second points is greater than that between the second and third, even though, in fact, it may be physically equal or less. A few years later, Geldreigh (1934) demonstrated an analogous effect in visual perception, and Bill and Teft (1969) confirmed this visual tau effect.

Meanwhile, Cohen, Hansel, and Sylvester (1954), using the method of adjustment, found an effect similar to the tau effect in bisection judgments for pitch and labeled it the "auditory tau effect." If a subject hears, through headphones, a repeated succession of three different tones

This research was supported in part by a Grant-in-Aid for Scientific Research, Ministry of Education, Science and Culture (No. 60710083), 1985. The author wishes to thank Professor Tadasu Oyama of the Department of Psychology, University of Tokyo, for his encouragement and helpful suggestions during the course of this work and in the preparation of the manuscript. The author is indebted to Professors Shigeru Kiritani, Masayuki Sawashima, and Hajime Hirose of the Research Institute of Logopedics and Phoniatrics, Faculty of Medicine, University of Tokyo, for making facilities available for the preparation of the stimuli.

The author's mailing address is: Kitasato University, College of Liberal Arts and Sciences, 1-15-1 Kitasato, Sagamihara-shi, Kanagawa, 228 Japan. of brief and equal duration and is asked to adjust the middle tone to appear to be intermediate in pitch between the other two tones, when the tone is temporally close to either of the two comparison tones it is judged as more different in frequency than when it is more temporally distant. Cohen, Christensen, and Ono (1974) found that this auditory tau effect occurs monaurally as well as binaurally, and they proposed a "high level" model with a hierarchical order of perceptual processes into which temporal and/or spatial factors enter as an integral component. However, they proposed this model "in the most tentative fashion, since so much of the neurophysiological basis of the various effects remains obscure"' (p. 86). The influence of temporal factors upon the hierarchical character, in particular, has not been fully examined.

Christensen and Huang (1979) examined the auditory tau effect using the method of adjustment with a more precise control of the time condition, and reported that the findings were in perfect accordance with those reported by Cohen et al. (1974) and Cohen et al. (1954). Christensen and Huang (1979) offered an explanation for the auditory tau effect in terms of memory for pitch in which the dynamic role of the time condition is taken into account. According to their model, on the first presentation of the three tones, the subject has to compute the midpoint $\left(T 2^{\prime}\right)$ of the first tone (T1) and the third tone $(T 3)$ by means of some algorithm. Since $T 2^{\prime}$ (the subjective middle frequency) did not arise from direct sensory input, it can be hypothesized that it is stored in a manner that renders it proof against interference by incoming tones but not against the decay of memory. On the second presentation of the cycle, the second tone $(T 2)$ can be compared with $T 2^{\prime}$ as soon as the second tone (T2) is avail- 
able, and the trial is over if they are judged to be the same. If they are not, then $T 2$ ' can be 'refreshed" by another calculation and the process repeated, $T 2$ on the $n$th trial being compared with $T 2^{\prime}$ derived from the $(n-1)$ th trial. In order to compute $T 2^{\prime}, T 1$ must be held in memory until $T 3$ arrives, by which time $T 2$ will have been presented. Christensen and Huang (1979) further supposed that the effect of the interference of $T 2$ with $T 1$ was to make the retrieved value of $T 1$ appear closer in pitch to $T 2$ than was physically the case. Thus, in ascending order of pitch, $T 2$ ' would have a larger (higher) value than would otherwise be the case (see discussion in Christensen \& Huang, 1979).

In order to elucidate the mechanism of the auditory tau effect, it is necessary to explore some questions which remain unanswered. First, the mechanism of the auditory tau effect is often explained by factors that greatly depend upon the experimental procedure. In many previous investigations on this topic (e.g., Cohen et al., 1974), the method of adjustment was used. According to this method, it is necessary to keep in memory the subjective middle frequency between the first and third tones until the second stimulus of the next triad appears, so the auditory tau effect might be based on the interaction of memories of more than three stimuli. Consequently, it would be implausible to regard the "auditory tau effect" as an example of the tau effect. The underlying central mechanism of the auditory tau effect obtained from pitch bisection judgments would seem to be different from those of the tau effect in other sensory domains.

Second, the relationship between the auditory tau effect and stimulus attributes remains unexamined. While the auditory tau effect has been taken as an example of the interdependence of time-pitch perception in pitch bisection experiments, phenomena similar to the auditory tau effect have also been investigated from other points of view. For example, time error (Köhler, 1923) is another phenomenon caused by the effect of temporal variables upon pitch judgments. When two stimuli equal in value are presented in temporal succession, there exists a constant error in comparative judgment for the two stimuli as a function of the lapse of time between the first and second. It has been shown that if the target is presented second, the judgments show systematic tendencies toward either overestimation or underestimation of pitch, depending on temporal conditions. Time error seems to be very similar to the auditory tau effect. It has been found that the direction and magnitude of time error vary systematically both with the temporal interval between two stimuli and with the attributes of the stimuli (Morikiyo, 1959; Postman, 1946). In order to understand the basic mechanism of the auditory tau effect, it is also necessary that the relationship between the auditory tau effect and stimulus attributes should be investigated experimentally.

On the other hand, additional research into time-space relations in perception has focused on the effect of variations in the visual space on perceived temporal durations.
Abbe (1936) has shown that when two equal time intervals are separated by stimuli demarcating different spatial extents, stimuli with a larger spatial extent result in a longer perceived duration of the temporal interval. Similar results have been obtained by Cohen, Hansel, and Sylvester (1953), who labeled their finding the "kappa effect" to distinguish it from the tau effect, and by Price-Williams (1954). The kappa effect has also been shown to occur in various sense modalities, including vision, audition, and touch. It appears that some investigations of the interdependence of time and space have shared common experimental conditions for both the tau effect and the kappa effect. The investigations on the tau and kappa effects suggest that there is a tradeoff between time and space perceptions.

In the present study, three experiments were conducted. The purpose of the first experiment, which was preliminary in nature, was to replicate the basic finding of Cohen et al. (1954) that the auditory tau effect occurs in pitch perception, although the experiment also addressed the question of whether the auditory tau effect is produced by an $A X B$ paradigm. The aims of the second and third experiments were to investigate the underlying central mechanisms of the auditory tau and kappa effects, respectively. The second experiment focused on the relationship between the auditory tau effect and stimulus attributes. The third experiment focused on the relationship between the auditory kappa effect and stimulus attributes.

\section{EXPERIMENT 1}

The purpose of Experiment 1 was to investigate whether the auditory tau effect in the pitch perception of pure tones could be obtained in ratings of similarity, which are most commonly used in auditory perceptual experiments. In this experiment, an $A X B$ paradigm was used to elicit similarity ratings from subjects. According to this paradigm, subjects hear one triad and have only to judge whether the second stimulus is similar to the first or to the third. Since the auditory tau effect obtained from pitch bisection judgments using the method of adjustment might be based on the interaction of memories of more than three stimuli, an $A X B$ paradigm might be superior to the method of adjustment in inducing performance that would more directly reflect the effect of temporal variables upon the pitch perception of the second tone. Experiment 1 therefore used an $A X B$ paradigm with short ISIs instead of the method of adjustment.

\section{Method}

Subjects. The subjects were 7 undergraduate students at Chiba University. None reported any history of a speech or hearing disorder.

Stimuli. Sine-wave tones, which were prepared using a PDP-11 computer (DEC), were normalized using the overall maximum amplitude. All the stimuli were read out at a sampling rate of $10 \mathrm{kHz}$ with an accuracy of 10 bits, converted into analog waveform, and recorded on magnetic tape through a low-pass filter with a cutoff 


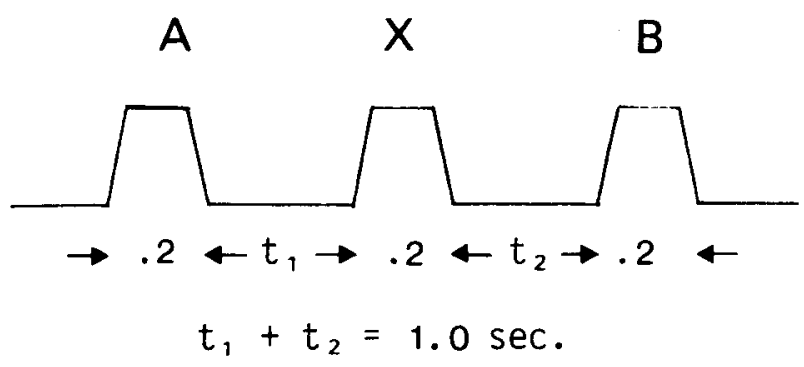

Figure 1. Presentation of stimuli in an $A X B$ paradigm.

frequency of $4.5 \mathrm{kHz}$ for the off-line experiment. All the stimuli had a steady duration of $200 \mathrm{msec}$, with rise and decay times of $20 \mathrm{msec}$.

One stimulus set consisted of three tones. The frequencies of the first and the third tones were held constant at 1000 and $2500 \mathrm{~Hz}$, respectively. The frequency range of the second tone was from 1350 to $2150 \mathrm{~Hz}$, and nine stimuli were so selected as to divide the frequency range into eight equal intervals. The step size between adjacent stimuli was $100 \mathrm{~Hz}$. Thus, nine types of stimulus triad were formed. Successive trials were separated by $3 \mathrm{sec}$, and there was a 5 -sec pause after every 10 trials.

Procedure. To study the auditory tau effect, an $A X B$ method was used instead of the method of adjustment. As shown in Figure 1, three tones- $A, X$, and $B$-were presented successively. The subjects were required to judge whether the pitch of the second (target) tone $(X)$ was more similar to that of $A$ or $B$. Two successive short time intervals, represented as $t 1$ (between $A$ and $X$ ) and $t 2$ (between $X$ and $B$ ), were delimited by the three tones and were varied by changing the temporal position of the second tone. Five temporal conditions, represented by the time ratio $t 1 /(t 1+t 2)$, were $.05, .25, .50, .75$, and .95 . The total duration $(t 1+t 2)$ remained constant at $1.0 \mathrm{sec}$.

The subjects were given a randomized sequence of 100 triads five times. The initial five and the last five trials of each series were excluded from the data. The total number of trials was 500 for each subject $\{[(5$ temporal conditions $\times 9$ target stimuli $\times 2)+10 \mathrm{ex}-$ tra trials] $\times 5\}$. Ten judgments for each of the nine targets were obtained from each subject for each of the five temporal conditions.

The recorded stimuli were played back at a comfortable listening level on a tape recorder (Sony, TC-365).

\section{Results and Discussion}

The probability that the target tone would be judged as similar to either one of the two other tones as a function of the tone frequency can be generally approximated by a cumulative normal distribution. Therefore, the percentage of responses indicating that the target was similar to the third tone for the five temporal conditions for each subject was approximated by a cumulative normal distribution whose mean and standard deviation were obtained by a normal graphic process for each subject, as outlined by Guilford (1954). The mean indicates the subjective middle frequency between the first and third tones and theoretically corresponds to the mean adjusted tone frequency by the method of adjustment (e.g., Christensen \& Huang, 1979). The standard deviation serves as an index for the accuracy of the judgments. The subjective middle frequency and standard deviation for each subject under these conditions are given in Table 1. The data essentially replicate the findings obtained in previous studies, even though the method of adjustment was replaced by the $A X B$ method. The difference in the means between the time ratio conditions of .05 and .95 was found to be significant by $t$ test $[t(5)=4.319, p<.01]$.

The mean (subjective middle frequency) obtained from the pooled data of all the subjects was calculated by the method of maximum likelihood with Müller-Urban weight and plotted as a function of the time ratio condition of the second tone in Figure 2. The subjective middle frequency decreased as the second tone came temporally closer to the third tone. This result can be understood to reflect the fact that when the second tone came temporally closer to the third (the highest) tone, the subjects perceived the second tone as being higher than its actual frequency, and, thus, the subjective middle frequency was lowered. When the second tone was put closer to the first (the lowest) tone, on the other hand, the subjects perceived the second tone as being lower than its actual frequency, and, thus, the subjective middle frequency was raised. Accordingly, Figure 2 shows a downward linear trend.

The findings obtained in Experiment 1 show that the explanation offered by Christensen and Huang (1979) for the mechanism of the auditory tau effect is inadequate in certain respects. They explained the mechanism of the auditory tau effect in pitch bisection judgments by supposing that the interference of $T 2$ with $T 1$ made the retrieved value of $T 1$ appear closer in pitch to $T 2$ than the actual frequency of $T 1$, and that then $T 2^{\prime}$ (the subjective middle frequency) would be estimated as being closer to $T 3$. $T 2$ on the $n$th trial was compared with $T 2^{\prime}$ derived from the $(n-1)$ th trial. The results of the present experiment, however, cannot be explained by this hypothesis, because under an $A X B$ paradigm the subjects judge whether the pitch of the second tone $(X)$ is more similar to $A$ or $B$ as

Table 1

The Subjective Middle Frequency and Standard Deviation (in Hertz) for Each Subject

\begin{tabular}{|c|c|c|c|c|c|c|c|c|c|c|}
\hline \multirow{3}{*}{$\begin{array}{c}\text { Subject } \\
\text { Number }\end{array}$} & \multicolumn{10}{|c|}{ Value of $t_{1} /\left(t_{1}+t_{2}\right)$} \\
\hline & \multicolumn{2}{|c|}{.05} & \multicolumn{2}{|c|}{.25} & \multicolumn{2}{|c|}{.50} & \multicolumn{2}{|c|}{.75} & \multicolumn{2}{|c|}{.95} \\
\hline & Mean & $S D$ & Mean & $S D$ & Mean & $S D$ & Mean & $S D$ & Mean & $S D$ \\
\hline 1 & 1893 & 257 & 1825 & 251 & 1833 & 221 & 1737 & 256 & 1690 & 226 \\
\hline 2 & 1840 & 269 & 1812 & 269 & 1821 & 230 & 1741 & 218 & 1747 & 206 \\
\hline 3 & 1630 & 273 & 1713 & 252 & 1767 & 254 & 1675 & 287 & 1464 & 368 \\
\hline 4 & 1962 & 392 & 1900 & 450 & 1809 & 331 & 1728 & 245 & 1483 & 335 \\
\hline 5 & 1754 & 399 & 1706 & 272 & 1685 & 250 & 1661 & 253 & 1565 & 338 \\
\hline 6 & 1893 & 257 & 1825 & 251 & 1833 & 221 & 1737 & 256 & 1690 & 226 \\
\hline Mean & 1829 & & 1797 & & 1791 & & 1713 & & 1607 & \\
\hline
\end{tabular}




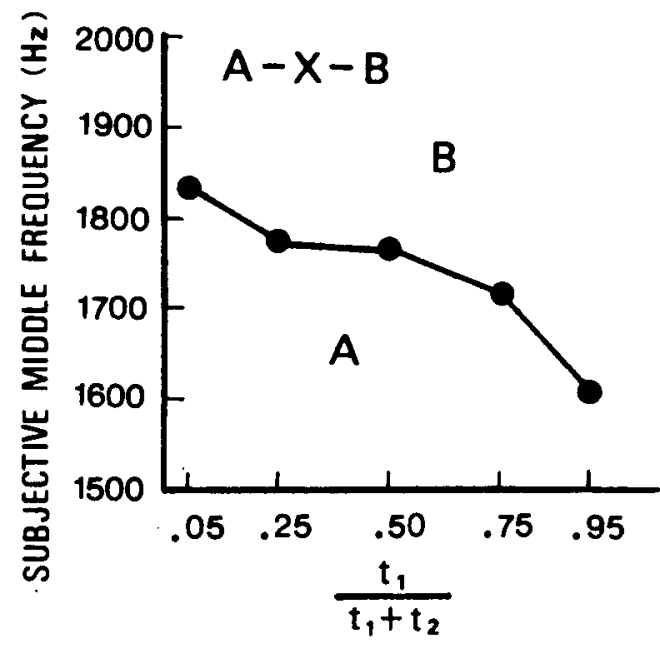

Figure 2. The subjective middle frequency (mean frequency) in hertz of the pooled data of all the subjects as a function of the time ratio condition of the second (target) tone.

soon as they hear the triad, and they do not need to keep the pitch of the second tone in memory beyond one triad. Nor do they need to estimate the subjective middle frequency. It might be said that the hypothesis of Christensen and Huang (1979) greatly depends upon the experimental situation.

Another possible explanation for the results of Experiment 1 , then, is that the auditory tau effect is caused by the integration of a forward context effect (the influence of the first stimulus on the perception of the second, target stimulus) and a backward context effect (the influence of the third stimulus on perception of the second, target stimulus) within one triad. The differences in magnitude between the forward and backward context effects upon the perception of the second tone might be an essential factor in the auditory tau effect. It has been reported that context effects expressed in terms of a shift in category boundary show a continuous transition from assimilation to contrast, and that contrast becomes more intense with increased stimulus complexity (Shigeno \& Fujisaki, 1979). If the auditory tau effect is a context effect, its occurrence should depend upon stimulus complexity.

\section{EXPERIMENT 2}

The purpose of Experiment 2 was to examine whether the auditory tau effect could be produced when a stimulus attribute more complex than the pitch of pure tones was used. It has been reported that a phenomenon similar to the auditory tau effect can be obtained in two-tone (or two-sound) situations in the perception of speech and nonspeech stimuli (e.g., Shigeno \& Fujisaki, 1979). Either assimilation or contrast is obtained as a context effect in these situations. Assimilation is a shift of category boundary in the direction opposite to that of the context stimulus. Contrast, on the other hand, is a shift of category boundary toward the context stimulus. Since the auditory tau effect found in pitch bisection judgments is defined as the phenomenon that occurs when the subjective mid- dle frequency between the first and third stimuli moves in the direction opposite to that of the temporally closer stimulus (Cohen et al., 1954), the auditory tau effect might be explained in terms of assimilation.

Shigeno and Fujisaki (1980) investigated the context effects in two-sound situations using vowels and found that assimilation occurred when subjects were required to respond in nonphonetic (pitch of vowels) judgments, whereas contrast occurred when subjects were required to respond in phonetic (phoneme of vowels) judgments. The influence of the mode of judgment-phonetic vs. nonphonetic-upon the auditory tau effect has not been investigated. In this experiment, the question of whether or not the auditory tau effect occurs when subjects are asked to respond in phonetic judgments was examined.

\section{Method}

Subjects. The subjects were 19 undergraduate students at Kitasato University. None reported any history of a speech or hearing disorder. All were native speakers of Japanese.

Stimuli. Synthetic vowels were prepared using a PDP-11 computer (DEC). These were generated by a terminal-analog speech synthesizer and were normalized by the overall maximum amplitude. All the stimuli were read out at a sampling rate of $10 \mathrm{kHz}$ with an accuracy of 10 bits, converted into analog waveform, and recorded through a low-pass filter with a cutoff frequency of $4.5 \mathrm{kHz}$ on a magnetic tape for the off-line experiment. All the stimuli had a steady duration of $200 \mathrm{msec}$ with rise and decay times of $20 \mathrm{msec}$.

Four stimulus continua of speech sounds $(/ \mathrm{e} /-/ \mathrm{i} /, / \mathrm{i} /-\mathrm{e} /, / \mathrm{I} /-\mathrm{i} /$, and $/ \mathrm{i} /-/ \mathrm{I} /$ ) were used in this experiment. In Japanese, $/ \mathrm{e} / \mathrm{and} / \mathrm{i} /$ are perceived to be typical vowels; the sound between $/ \mathrm{i} /$ and $/ \mathrm{e} /$ is perceived to be ambiguous, and is designated here as /I/. According to a previous study (Fujisaki, Nakamura, \& Yoshimune, 1970 ), the sounds / $/$ and $/ \mathrm{i} /$ belong to different phonetic categories whereas $/ \mathrm{I} /$ and $/ \mathrm{i} /$ belong to the same phonetic category in an average speaker of Japanese. Thus, it can be said that the stimulus continua $/ \mathrm{e} / \mathrm{-} / \mathrm{i} / \mathrm{and} / \mathrm{i} / \mathrm{-} / \mathrm{e} / \mathrm{consist}$ of "between-phonetic-category" stimuli, and that the stimulus continua $/ \mathrm{I} / \mathrm{-} / \mathrm{i} /$ and $/ \mathrm{i} /-/ \mathrm{I} /$ consist of "within-phonetic-category" stimuli. The first formant frequencies of $/ \mathrm{e} /, / \mathrm{i} /$, and $/ \mathrm{I} /$ were 520,270 , and $370 \mathrm{~Hz}$, respectively. The other stimulus parameters of these vowels are shown in Table 2.

Table 2

Stimulus Parameters (in Hertz) Used in Experiment 2

\begin{tabular}{rccc}
\hline Stimulus & \multicolumn{3}{c}{ Formant Frequencies } \\
\cline { 2 - 4 } Number & $\mathbf{F}_{1}$ & $\mathrm{~F}_{2}$ & $\mathbf{F}_{3}$ \\
\hline ii/ 1 & 300 & 2500 & 2800 \\
2 & 350 & 2360 & 2760 \\
3 & 370 & 2310 & 2740 \\
4 & 390 & 2260 & 2720 \\
5 & 410 & 2210 & 2700 \\
6 & 430 & 2160 & 2680 \\
7 & 450 & 2110 & 2660 \\
8 & 470 & 2060 & 2640 \\
/e/ 9 & 520 & 1920 & 2600 \\
& & & \\
ii $/ 10$ & 270 & 2140 & 2780 \\
11 & 300 & 2140 & 2780 \\
12 & 310 & 2140 & 2780 \\
13 & 320 & 2140 & 2780 \\
14 & 330 & 2140 & 2780 \\
15 & 340 & 2140 & 2780 \\
16 & 350 & 2140 & 2780 \\
17 & 360 & 2140 & 2780 \\
/I/ 18 & 390 & 2140 & 2780 \\
\hline
\end{tabular}


Each stimulus set consisted of three scunds. When the first sound was /e/ and the third sound was /i/, one of seven stimuli on the $/ \mathrm{e} /-\mathrm{i} / \mathrm{stimulus}$ continuum was selected as the second (target) sound in each trial. The first formant frequencies (F1s) of all seven sounds formed a continuum between 470 and $350 \mathrm{~Hz}$ with a step size of $20 \mathrm{~Hz}$. When the first sound was /i/ and the third sound was /e/, on the other hand, the F1s of the second sound formed a continuum between 350 and $470 \mathrm{~Hz}$ with a step size of $20 \mathrm{~Hz}$. The second and the third formant frequencies $(F 2, F 3)$ of these sounds were varied linearly on the $/ \mathrm{e} / \mathrm{-} / \mathrm{i} /$ or $/ \mathrm{i} /-\mathrm{e} / \mathrm{e}$ continuum to give a stronger identity to these vowels. In the case of the $/ \mathrm{I} /-/ \mathrm{i} /$ and $/ \mathrm{i} /-/ \mathrm{I} /$ continua, the F1s of the target sound varied from 360 to $300 \mathrm{~Hz}$ and from 300 to $360 \mathrm{~Hz}$, respectively. The step size was $10 \mathrm{~Hz}$. F2 and F3 were fixed at 2140 and $2780 \mathrm{~Hz}$, respectively. The fundamental, fourth, and fifth formant frequencies (F0, F4, F5) of all stimuli were held constant at 140,3500 , and $4500 \mathrm{~Hz}$, respectively. The bandwidths of F1, F2, F3, F4, and F5 were also held constant at $60,100,120,175$, and $280 \mathrm{~Hz}$, respectively.

Procedure. The subjects were divided into four groups, of 6 subjects each, according to the four stimulus continua: $/ \mathrm{e} /-/ \mathrm{i} /, / \mathrm{i} /-\mathrm{e} /$, $/ / /-/ i /$, and $/ i /-/ I /$. Five of the 19 subjects participated in two of the four conditions.

The $A X B$ method was employed. The subjects were required to judge whether $X$ was similar to $A$ or to $B$. The temporal positions of the second sound $(X)$, represented as the time ratio $t 1 /(t 1+t 2)$, were varied under the same five conditions as in Experiment 1. The total duration $(t 1+t 2)$ remained constant at $1.0 \mathrm{sec}$.
The subjects were twice given the same series consisting of 150 triads. The initial five and the last five trials of each series were excluded from the data. The total number of trials was 300 for each subject $\{[(5$ temporal conditions $\times 7$ target stimuli $\times 4)+10$ extra trials] $\times 2\}$. Eight judgments for each target sound were obtained from each subject for each of the five temporal conditions.

The recorded stimuli were played back on a tape recorder (National, RU-2006u) and were presented to the subjects in a quiet room through a loudspeaker (National, WS320) at a comfortable listening level.

\section{Results and Discussion}

The means and standard deviations obtained by a normal graphic process for each subject are given in Table 3, where the mean represents the category boundary between $/ \mathrm{e} /$ and $/ \mathrm{i} /$ or between $/ \mathrm{I} /$ and $/ \mathrm{i} /$. The differences in these category boundaries due to the time ratio $t 1 /(t 1+t 2)$ were found to be significant by a $t$ test between the time ratio conditions .05 and .95 [for the $/ \mathrm{e} /-/ \mathrm{i} /$ continuum, $t(5)=$ $4.89, p<.01$; for the $/ \mathrm{i} /-/ \mathrm{e} /$ continuum, $t(5)=2.69$, $p<.05]$. Likewise, the differences in the category boundaries of the $/ \mathrm{I} /-/ \mathrm{i} /$ and $/ \mathrm{i} /-/ \mathrm{I} /$ continua between the time ratio conditions .05 and .95 were found to be significant by a $t$ test [for the $/ \mathrm{I} / \mathrm{-} / \mathrm{i} /$ continuum, $t(5)=7.633$,

Table 3

The Subjective Middle F1 and Standard Deviation (in Hertz) for Each Subject

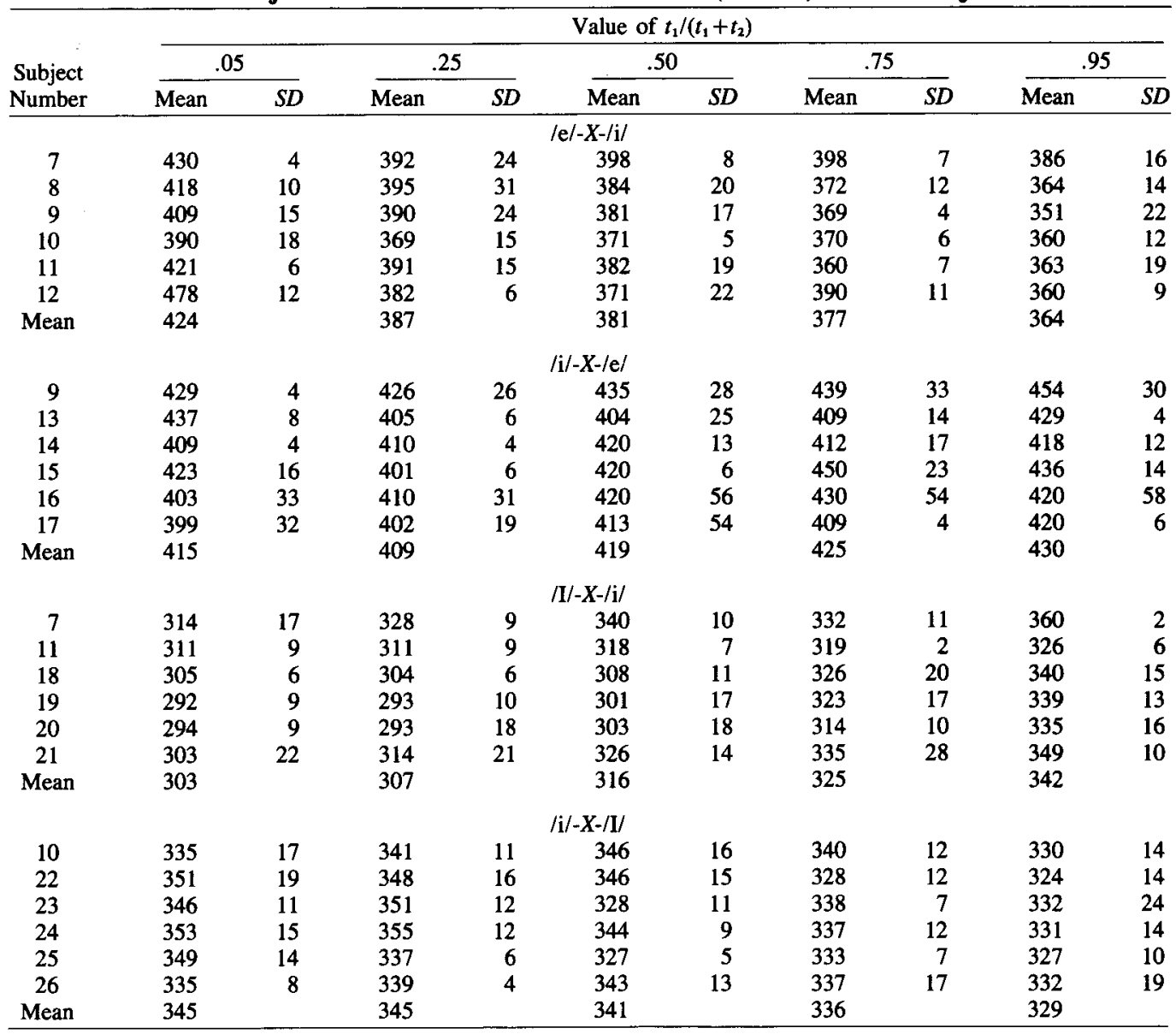


$p<.01 ;$ for the $/ \mathrm{i} /-/ \mathrm{I} /$ continuum, $t(5)=3.85$, $p<.05]$.

The mean (subjective middle F1) obtained from the pooled performance of all the subjects was calculated by the method of maximum likelihood with Müller-Urban weight and plotted in Figure 3 as a function of the time ratio condition of the second sound. The subjective middle F1 corresponds to the subjective phonetic category boundary between /e/ and /i/ or the subjective responsecategory boundary between $/ \mathrm{I} / \mathrm{and} / \mathrm{i} / \mathrm{\text {.Theuppergraphs }}$ show the results for the $/ \mathrm{e} /-/ \mathrm{i} / \mathrm{and} / \mathrm{i} /-\mathrm{e} / \mathrm{stimulus}$ continua; the lower graphs show the results for the $/ \mathrm{I} /-/ \mathrm{i} /$ and /i/-/I/ stimulus continua.

In the case of the $/ \mathrm{e} /-\mathrm{i} /$ and $/ \mathrm{i} / \mathrm{-} / \mathrm{e} / \mathrm{continua}$ (the upper panels of Figure 3), the subjective middle F1 (the phonetic category boundary between $/ \mathrm{e} /$ and $/ \mathrm{i} /$ ) moves toward the sound that is temporally closer to the second sound. For example, in the left upper panel, the interpolated subjective middle $F 1$ decreases as the time ratio increases. This indicates that the percentage of responses in which the second sound is judged to be similar to the third sound (/i/) diminishes as the second sound becomes temporally closer to the third sound. Similar results in the opposite direction were obtained with the /i/-/e/ continuum (the right upper panel), although the effects were less than those in the case of the /e/-/i/ continuum.

In the case of the $/ \mathrm{I} /-/ \mathrm{i} /$ and $/ \mathrm{i} /-/ \mathrm{I} /$ continua (the lower panels of Figure 3), on the other hand, the subjective middle F1 (the response-category boundary between /I/ and /i/) moves opposite to the sound that is temporally closer to the second sound. For example, in the left lower panel, the interpolated subjective middle $F 1$ increases as the time ratio increases. This means that the percentage of responses in which the second sound is judged to be similar to the third sound $(/ \mathrm{i} /)$ increases as the second sound becomes temporally closer to the third sound. Similar results in the opposite direction were obtained with the /i/-/I/ continuum (the right lower panel), although the effects were less than those for the $/ \mathrm{I} /-/ \mathrm{i} /$ continuum.

It is thus possible to say that, in speech perception, the auditory tau effect does not occur when the stimuli are between different phonetic categories, such as /e/ and /i/, although it does occur, as for pure tones, when the stimuli are within the same phonetic category, such as /I/ and
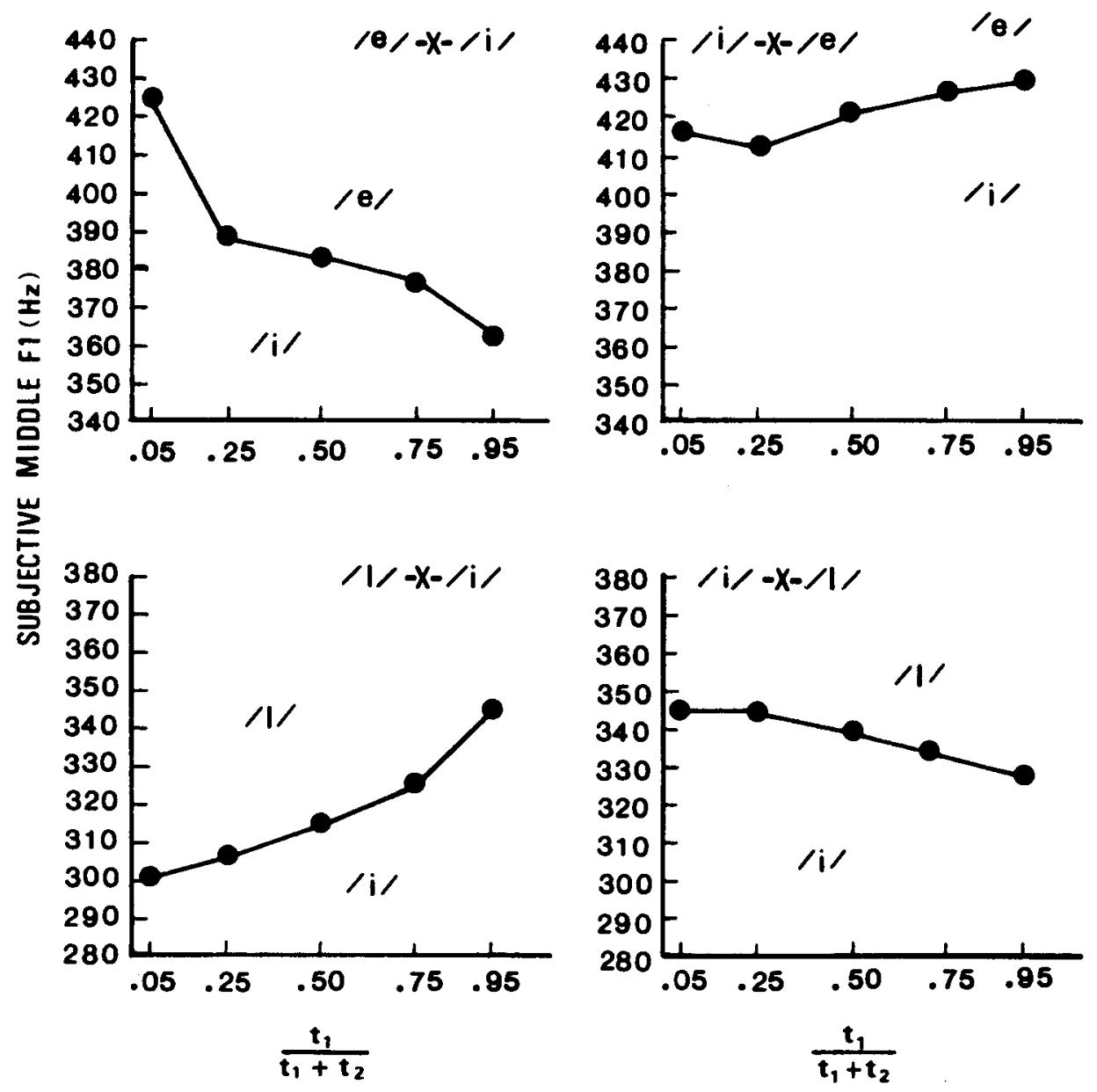

Figure 3. The subjective middle F1 (mean F1) in hertz of the pooled performances of all the subjects as a function of the time ratio condition of the second sound. 
/i/. These results suggest that whether the stimulus is speech or nonspeech is not an essential factor for the auditory tau effect, but that whether the stimulus continuum consists of two different phonetic categories or of the same phonetic category (or pitch of pure tones) is an essential factor.

Furthermore, the auditory tau effect can be understood as a context effect, since the shift of category boundary (or the shift of the subjective middle frequency) in the opposite direction (i.e., assimilation) to that of the temporally closer stimulus seems to be an essential component in the auditory tau effect. Shigeno and Fujisaki (1979) found that the context effect shows a continuous transition from assimilation to contrast as the temporal/spatial distance between the context and the target stimuli is increased, although the contrastive effect becomes more intense with increased stimulus complexity. Shigeno and Fujisaki (1979) assumed that the context effect consisted of two components with opposite signs and different decay rates: a fast-decaying assimilative component at the precategorical level and a slowly decaying contrastive component at the categorical level. Following Fujisaki and Kawashima (1971), Shigeno and Fujisaki (1979) further claimed that these two components might be associated with two distinct short-term storage systems: the precategorical (Crowder \& Morton, 1969; Pisoni, 1975) and the categorical (see discussion in Shigeno \& Fujisaki, 1979). According to this model, the categorical judgment for a target stimulus is based on the category boundary stored in long-term memory, but is influenced by information on the preceding and following context stimuli temporarily retained in the two short-term memories. Assimilation and contrast may be ascribed to the differences in the relative magnitude of the two short-term memories. It might be said that the auditory tau effect consists mainly of the assimilative component at the auditory (precategorical) level.

Figure 3 also shows that the two upper panels and the two lower panels are asymmetrical to each other. These results for speech stimuli are very different from the data obtained by Cohen et al. (1974), who found a strikingly smooth and symmetrical pattern for the ascending and descending orders in the case of the auditory tau effect for tonal intervals. Other examples of the discrepancy between speech and nonspeech perception (or phonetic and nonphonetic judgments) is provided by the pattern of forward and backward context effects. Repp, Healy, and Crowder (1979) and Shigeno and Fujisaki (1980) found that backward contrast was stronger than forward contrast in phonetic judgments of isolated synthetic vowels. Shigeno and Fujisaki (1980) found that nonphonetic judgments (pitch of vowels) of isolated synthetic vowels exhibited smaller contrast effects and no (or the opposite) difference between forward and backward contrast. As for contextual contrast in vowel identification by anchoring, Sawusch, Nusbaum, and Schwab (1980) observed contrast from both ends of the /i/-/I/ continuum, which they assigned to two different mechanisms. Crowder and
Repp (1984), on the other hand, found an asymmetry in contrast across both the $/ \mathrm{i} /-/ \mathrm{I} /$ and $/ \varepsilon /-/ æ /$ continua, since no contrast was obtained when each target was preceded by one continuum endpoint and abundant contrast was obtained when each target was preceded by the other endpoint. The magnitude of the forward and backward context effects may vary with the vowel categories used as context stimuli and may cause the asymmetry of the auditory tau effect.

Taking these results into consideration, it might be possible to define the auditory tau effect in vowel perception in terms of the differences in the magnitudes of forward and backward context effects for vowels. It has also been hypothesized that the contrastive component should be greater in phonetic judgments than in nonphonetic judgments (Shigeno \& Fujisaki, 1980). In the case of the /e/$/ \mathrm{i} /$ and $/ \mathrm{i} /$-/e/ continua, a slowly decaying contrastive component would be more influential than a fast-decaying assimilative component, because /e/ and /i/ are phonetically distinct from each other. When the second sound is temporally closer to the third sound, the backward contrastive context effects of the third sound are considered to be greater than the forward contrastive context effects of the first sound, so contrast with the third sound should occur (i.e., the category boundary should move toward the third sound). When the second sound is temporally closer to the first sound, on the other hand, the forward contrastive context effects of the first sound are considered to be greater than the backward contrastive context effects of the third sound, so contrast with the first sound should occur (i.e., the category boundary should move toward the first sound). Meanwhile, in the case of the $/ \mathrm{I} /-/ \mathrm{i} /$ and $/ \mathrm{i} /-/ \mathrm{I} /$ continua, the contextual contrastive component should not be influential in either forward or backward context effect, because /i/ and /I/ are not phonetically distinct from each other for Japanese subjects. The contextual assimilative components at the precategorical level, on the other hand, are supposed to be available both in forward and backward context effects. When the second sound is temporally closer to the third sound, the backward assimilative context effects of the third sound can be expected to be greater than the forward assimilative context effects of the first sound, so assimilation to the third sound should occur (i.e., the category boundary should move in the direction opposite to that of the third sound). When the second sound is temporally closer to the first sound, on the other hand, the forward assimilative context effects of the first sound can be considered to be greater than the backward assimilative context effects of the third sound, so assimilation to the first sound should occur (i.e., the shift of category boundary should move in the direction opposite to that of the first sound).

\section{EXPERIMENT 3}

The purpose of Experiment 3 was to explore further aspects of time-pitch (or time-phonetic category) relations 
in auditory perception. Since the relationship between stimulus attributes and time perception in the kappa effect had not yet been examined, the kappa effect, which has been considered to be the converse of the tau effect, was examined in Experiment 3 using speech and nonspeech stimuli.

\section{Method}

Subjects. The subjects were 20 undergraduate students at Kitasato University. None reported any history of a speech or hearing disorder. All were native speakers of Japanese. Two had participated in Experiment 2.

Stimuli. Sine-wave tones and synthetic vowels were used. These were essentially the same as those used in Experiments 1 and 2. In the case of the pure tones, the first and the third stimuli were fixed at 1000 and $2500 \mathrm{~Hz}$, respectively; the second stimulus was $1000,1350,1750,2150$, or $2500 \mathrm{~Hz}$. In the case of the synthetic vowels, the first and the third stimuli were /i/ and /e/, respectively. These two vowels belong to different phonetic categories in Japanese. The F1 of the second stimulus was $300,350,410,470$, or $520 \mathrm{~Hz}$.

Procedure. The subjects were divided into two groups. Each group consisted of 10 subjects.

The $A X B$ method was employed. In this experiment, the subjects were asked to judge whether the second (target) stimulus was closer to $A$ or to $B$ in time, not in pitch. The interstimulus intervals between the first and second and between the second and third stimuli were $t 1$ and $t 2$, respectively. Seven temporal conditions, represented by the time ratio $t 1 /(t 1+t 2)$, were $.41, .44, .47, .50, .53, .56$, and .59. The total duration $(t 1+t 2)$ was constant at $1.0 \mathrm{sec}$.
The subjects in each group were twice given the same stimulus series consisting of 150 triads. The initial five and the last five trials of each stimulus series were excluded from the data. The total number of trials was 300 for each subject $\{[(5$ variables of the frequency (or F1) of the second stimulus $\times 7$ temporal conditions $\times 4$ ) + 10 extra trials] $\times 2$ \}. Eight judgments for each second stimulus were obtained from each subject for each of the five frequency (or F1) conditions of the second stimulus.

The recorded stimuli were played back on a tape recorder (National, RU-2006u) and presented to the subjects in a quiet room through a loudspeaker (National, WS320) at a comfortable listening level.

\section{Results and Discussion}

The means and standard deviations obtained by a normal graphic process for each subject are given in Table 4, where the mean corresponds to the subjective middle point between the first and the third stimuli along the time axis. The difference in the subjective middle temporal points due to the two target stimulus conditions of 1000 and $2500 \mathrm{~Hz}$ was found to be significant by a $t$ test $[t(9)=$ $4.537, p<.01]$. The difference in the subjective middle temporal points due to the two target stimulus conditions of $/ \mathrm{i} /(\mathrm{F} 1=300 \mathrm{~Hz})$ and $/ \mathrm{e} /(\mathrm{F} 1=520 \mathrm{~Hz})$ was also found to be significant by a $t$ test $[t(9)=8.318$, $p<.01]$.

The subjective middle temporal point of the pooled performances of all the subjects was calculated by the method

Table 4

The Subjective Middle Temporal Point and Standard Deviation (in Milliseconds) for Each Subject

\begin{tabular}{|c|c|c|c|c|c|c|c|c|c|c|}
\hline \multirow{4}{*}{$\begin{array}{l}\text { Subject } \\
\text { Number }\end{array}$} & \multicolumn{10}{|c|}{ Pure Tone } \\
\hline & \multicolumn{10}{|c|}{ Frequencies of the Second Stimulus } \\
\hline & \multicolumn{2}{|c|}{$1000 \mathrm{~Hz}$} & \multicolumn{2}{|c|}{$1350 \mathrm{~Hz}$} & \multicolumn{2}{|c|}{$1750 \mathrm{~Hz}$} & \multicolumn{2}{|c|}{$2150 \mathrm{~Hz}$} & \multicolumn{2}{|c|}{$2500 \mathrm{~Hz}$} \\
\hline & Mean & $S D$ & Mean & $S D$ & Mean & $S D$ & Mean & $S D$ & Mean & $S D$ \\
\hline 9 & 571 & 21 & 490 & 65 & 493 & 94 & 490 & 47 & 420 & 39 \\
\hline 10 & 518 & 40 & 490 & 27 & 494 & 18 & 496 & 19 & 461 & 12 \\
\hline 27 & 494 & 50 & 515 & 30 & 491 & 25 & 474 & 40 & 467 & 33 \\
\hline 28 & 510 & 52 & 533 & 45 & 521 & 64 & 485 & 60 & 473 & 36 \\
\hline 29 & 493 & 46 & 487 & 43 & 458 & 33 & 452 & 75 & 455 & 76 \\
\hline 30 & 530 & 39 & 515 & 30 & 506 & 30 & 488 & 47 & 504 & 51 \\
\hline 31 & 493 & 38 & 521 & 45 & 500 & 15 & 467 & 27 & 435 & 28 \\
\hline 32 & 498 & 55 & 491 & 33 & 469 & 46 & 489 & 35 & 465 & 42 \\
\hline 33 & 506 & 29 & 510 & 32 & 508 & 28 & 491 & 28 & 465 & 30 \\
\hline 34 & 524 & 41 & 523 & 31 & 527 & 30 & 500 & 34 & 466 & 33 \\
\hline \multirow[t]{5}{*}{ Mean } & 514 & & 508 & & 497 & & 483 & & 461 & \\
\hline & \multicolumn{10}{|c|}{$\mid \mathrm{i} / \mathrm{X}-\mathrm{X} / \mathrm{e} /$} \\
\hline & \multicolumn{10}{|c|}{$F_{1}$ of the Second Stimulus } \\
\hline & \multicolumn{2}{|c|}{$300 \mathrm{~Hz}$} & \multicolumn{2}{|c|}{$350 \mathrm{~Hz}$} & \multicolumn{2}{|c|}{$410 \mathrm{~Hz}$} & \multicolumn{2}{|c|}{$470 \mathrm{~Hz}$} & \multicolumn{2}{|c|}{$520 \mathrm{~Hz}$} \\
\hline & Mean & $S D$ & Mean & $S D$ & Mean & $\overline{S D}$ & Mean & $\overline{S D}$ & Mean & $S D$ \\
\hline 35 & 533 & 35 & 523 & 37 & 497 & 36 & 467 & 47 & 475 & 28 \\
\hline 36 & 506 & 34 & 515 & 40 & 474 & 56 & 472 & 41 & 449 & 32 \\
\hline 37 & 533 & 51 & 517 & 73 & 473 & 90 & 467 & 54 & 446 & 48 \\
\hline 38 & 521 & 38 & 524 & 48 & 538 & 55 & 467 & 38 & 485 & 10 \\
\hline 39 & 557 & 37 & 560 & 45 & 521 & 35 & 490 & 27 & 462 & 32 \\
\hline 40 & 539 & 27 & 522 & 32 & 518 & 30 & 499 & 36 & 488 & 14 \\
\hline 41 & 500 & 30 & 511 & 22 & 482 & 26 & 502 & 6 & 482 & 16 \\
\hline 42 & 539 & 60 & 538 & 33 & 527 & 57 & 485 & 40 & 481 & 37 \\
\hline 43 & 548 & 42 & 530 & 63 & 480 & 62 & 452 & 50 & 470 & 40 \\
\hline 44 & 507 & 98 & 500 & 47 & 493 & 31 & 455 & 4 & 440 & 50 \\
\hline Mean & 528 & & 524 & & 500 & & 476 & & 468 & \\
\hline
\end{tabular}


of maximum likelihood with Müller-Urban weight and plotted as a function of the frequency (or F1) of the second (target) stimulus in Figure 4, where the two graphs represent the results for pure tones and synethtic vowels, respectively. In the case of pure tones (the upper panel of Figure 4), an examination of the curve shows that when the frequency of the second stimulus is lower than the geometric mean between the first and third $(1581 \mathrm{~Hz})$, the subjective middle temporal point is greater than the objective one; hence, the interval $t 1$ is perceived to be shorter than its actual temporal interval. When the frequency of the second stimulus approaches the geometric mean between the first and third, the perceived temporal interval $t 1$ approaches the actual temporal interval. When the frequency of the second stimulus is higher than the geometric mean between the first and third, the subjective middle temporal point is smaller; hence the temporal interval $t 1$ is perceived to be longer than the actual temporal interval. Similar results are obtained with synthetic vowels,

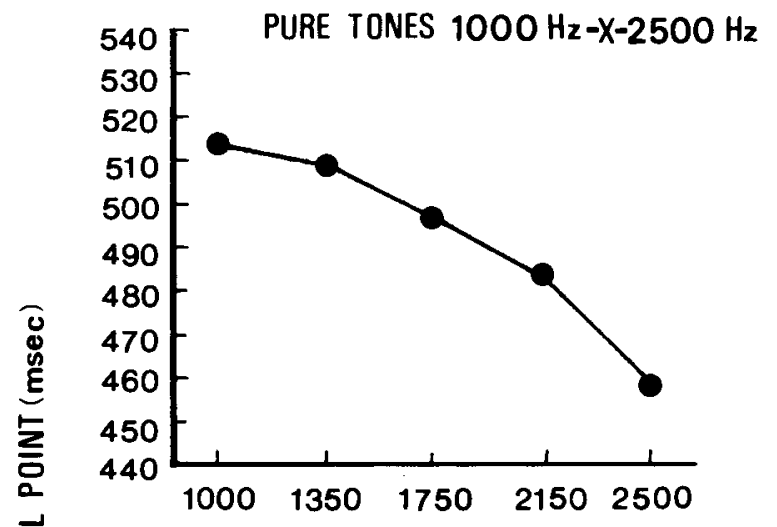

FREQUENCY OF SECOND (TARGET) STIMULUS $\left(\mathrm{Hz}_{\mathrm{z}}\right)$

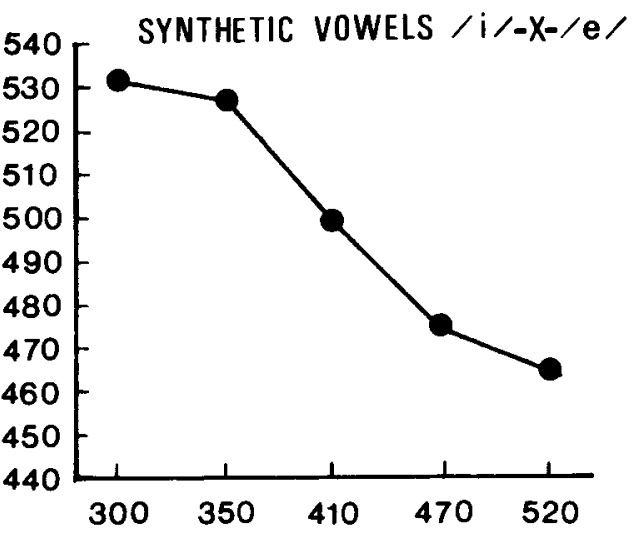

F1 OF SECOND (TARGET) STIMULUS $\left(\mathrm{Hz}_{\mathbf{z}}\right)$

Figure 4. The subjective middle temporal point in milliseconds of the pooled performances of all the subjects as a function of the frequency (or F1) of the second (target) stimulus. with which the same interpretation of the results can be presented (lower panel), although the geometric mean should be replaced by the physical middle F1 of the second stimulus $(410 \mathrm{~Hz})$. These results show that the kappa effect occurs for speech as well as nonspeech stimuli. That is, the kappa effect occurs irrespective of stimulus attributes.

Parks (1968) studied the kappa effect in vision and proposed that the kappa effect reflects not postperceptual processes, but a disturbance in the memory-trace of the temporal extent of the test interval. The results of Experiment 3 support these speculations, since the fact that both speech (phonetic) and nonspeech (nonphonetic) stimuli produce the kappa effect suggests that the kappa effect occurs at a common stage of auditory information processing before phonetic categorization.

\section{GENERAL DISCUSSION}

\section{Summary of Results}

The present study has four major results: (1) It provides evidence that the essential trend of results previously obtained using the method of adjustment with pure tones can be duplicated in an $A X B$ paradigm. (2) In speech perception, the auditory tau effect was observed to be significant when the stimulus continuum was $/ \mathrm{I} /-\mathrm{i} / \mathrm{h}$ or $/ \mathrm{i} /-/ \mathrm{I} /$, but the reverse pattern of results was obtained when the stimulus continuum was $/ \mathrm{e} /-/ \mathrm{i} /$ or $/ \mathrm{i} /-/ \mathrm{e} /$. (3) The results were not symmetrical between the /e/-/i/ and the $/ \mathrm{i} /-/ \mathrm{e} /$ continua, or between the $/ \mathrm{I} /-/ \mathrm{i} /$ and the $/ \mathrm{i} /-/ \mathrm{I} /$ continua. The results were observed more clearly for the $/ \mathrm{e} / \mathrm{-} / \mathrm{i} / \mathrm{con}-$ tinuum than for the $/ \mathrm{i} /-/ \mathrm{e} /$ continuum, and more clearly for the $/ \mathrm{I} /-/ \mathrm{i} /$ continuum than for the $/ \mathrm{i} /-/ \mathrm{I} /$ continuum. (4) The kappa effect occurred for both speech and nonspeech stimuli.

\section{The Auditory Tau Effect}

Previous researchers (Christensen \& Huang, 1979) offered an explanation for the auditory tau effect in terms of memory for pitch, and considered deterioration and interference in memory for pitch under the method of adjustment to be essential factors for this effect. The present study indicates that the mechanism of the auditory tau effect is not readily explained in terms of the deterioration and interference of memory for pitch, and shows that the explanations of previous researchers that depend on experimental procedure are inadequate for the reason that the same effect was observed under different procedures. The findings of the present study can be interpreted as suggesting that the auditory tau effect represents a context effect.

Several possible processing mechanisms for context effects have been proposed, particularly in connection with the contextual contrast effect. Sawusch and Nusbaum (1979) grouped possible mechanisms into three classes: feature detector fatigue, changes in response bias, and changes in the auditory ground. The feature-detector- 
fatigue hypothesis explains contextual effects in terms of the selective fatigue of a set of relative feature detectors. The response bias hypothesis (e.g., Parducci, 1975) suggests that vowel contrast effects are a product of changes in subjects' labeling strategies. These two hypotheses have been disputed, because they cannot adequately account for the adaptation/anchoring results found with vowels (see discussion in Sawusch \& Nusbaum, 1979). The hypothesis of changes in the auditory ground (Sawusch \& Nusbaum, 1979; Simon \& Studdert-Kennedy, 1978) suggests that contrast occurs when an auditory ground is established against which incoming stimuli are compared. Sawusch et al. (1980) suggest that contrast effects can be explained with reference to at least two different levels of processing: the auditory and the phonetic. For example, the effects of /i/ anchoring were to increase within/i/ category sensitivity, whereas /I/ anchoring shifted criterion placements. Recently, Crowder and Repp (1984) found that contrast was obtained even after F2 and F3 were removed from the stimulus that was preceded by the target and suggested a sensory, rather than a judgmental, basis for the vowel contrast effects. Fox (1985) found, however, that full-vowel anchors were more effective than either a single-formant anchor (only F1) or a pure-tone anchor in shifting subjects' identification of the test stimuli. He concluded that at least two different perceptual processes were involved in the production of vowel-contrast effects: the auditory and the phonetic.

Assimilation, on the other hand, has not been observed as often as contrast in perceptual judgments, and the mechanism of assimilation has received little explanation. Shigeno and Fujisaki (1979) found that the shift in category boundary caused by an anchor shows a continuous transition from assimilation to contrast as the temporal/spatial distance between the anchor and the target stimuli is increased. Repp (1978) also reported that the crossover points of the $\mathrm{CV}$ and VC functions change from assimilation to contrast with increasing closure duration. The present study indicates that assimilation seems to be an essential factor in the auditory tau effect, and suggests that the mechanism of the context effects in three-tone situations (including the auditory tau effect) can be described only in terms of a model that can explain the mechanisms of both contrast and assimilation (see Discussion, Experiment 2).

\section{The Kappa Effect}

Although the kappa effect has been regarded as the converse phenomenon of the tau effect, some differences in experimental results between the tau and the kappa effects were obtained in the present study. The major difference is that the tau effect greatly depends upon stimulus attributes, but the kappa effect is scarcely influenced by stimulus attributes or by judgmental modes-that is, phonetic or nonphonetic. This evidence strongly suggests that the kappa effect does not reflect a postperceptual process, but reflects the influence of the acoustic information of context stimuli upon the memory of the temporal intervals. Thus, the results suggest that the kappa effect occurs at a primary auditory level, and not at the judgmental level.

\section{Time-Space Interdependence}

The results of the present study on the auditory tau effect indicate that the hierarchical character of perceptual processes is influenced by temporal factors (temporal separation between two stimuli) at two different perceptual levels: the auditory (precategorical) and the phonetic (categorical). The results from the experiment on the kappa effect, on the other hand, show that time perception is influenced by spatial factors (spatial separation on the stimulus continuum between two stimuli) at the nonphonetic (precategorical) level, but not at the phonetic (categorical) level. The findings of the three experiments indicate the asymmetry of the time-space interaction.

To conclude, the present results have important implications for models of perceptual time-space interdependence in general, and suggest that the primary sensory (precategorical) level of hierarchical auditory information processing is involved in the production of these effects.

\section{REFERENCES}

AввE, M. (1936). The spatial effect upon the perception of time. Japanese Journal of Experimental Psychology, 3, 1-52.

Bill, J. C., \& TEFT, L. W. (1969). Space-time relations: Effects of time on perceived visual extent. Journal of Experimental Psychology, 81, 196-199.

Christensen, I. P., \& Huang, Y. L. (1979). The auditory tau effect and memory for pitch. Perception \& Psychophysics, 26, 489-494.

Cohen, J., Christensen, I. P., \& ONo, A. (1974). Influence of temporal intervals on comparative judgments of pitch: A study of subjective relativity. Tohoku Psychological Folia, 33, 76-87.

Cohen, J., Hansel, C. E. M., \& Sylvester, J. D. (1953). A new phenomenon in time judgment. Nature, 172, 901.

Cohen, J., Hansel, C. E. M., Sylvester, J. D. (1954). Interdependence of temporal auditory judgments. Nature, 174, 642.

Crowder, R. G., \& Morton, J. (1969). Precategorical acoustic storage (PAS). Perception \& Psychophysics, 5, 365-373.

Crowder, R. G., \& REPP, B. H. (1984). Single formant contrast in vowel identification. Perception \& Psychophysics, 35, 372-378.

Fox, R. A., (1985). Within- and between-series contrast in vowel identification: Full-vowel versus single-formant anchors. Perception \& Psychophysics, 38, 223-226.

FuJISAKI, H., \& KaWASHIMA, T. (1971). A model of the mechanisms for speech perception: Quantitative analysis of categorical effects in discrimination. Annual Report of the Engineering Research Institute, Faculty of Engineering, University of Tokyo, 30, 59-68.

Funsaki, H., Nakamura, N., \& Yoshimune, K. (1970). Analysis, normalization and recognition of sustained Japanese vowels. Joumal of the Acoustical Society of Japan, 26, 152-154. (In Japanese with English summary)

GELDREIGH, E. W. (1934). A lecture-room demonstration of the visual tau-effect. American Joumal of Psychology, 46, 483-485.

Gullford, J. P. (1954). Psychometric methods. New York: McGraw-Hill.

HeLson, H. (1930). The tau effect-An example of psychological relativity. Science, 71, 536-537.

KöHLER, W. (1923). Zur Theorie das Sukzessivvergleichs und der Zeitfehler. Psychologische Forschung, 4, 115-175.

MorIKIYO, Y. (1959). Time-order error in the successive comparison 
of tones: An examination by adaptation-level theory. Japanese Journal of Psychology, 30, 46-55. (In Japanese with English summary) PARducCI, A. (1975). Contextual effects: A range-frequency analysis. In E. C. Carterette \& M. P. Friedman (Eds.), Handbook of perception (Vol. 2). New York: Academic Press.

PARKs, T. E. (1968). The spatial separation of two light-flashes and their perceived separation in time. American Journal of Psychology, 81, 92-98.

PisonI, D. B. (1975). Auditory short-term memory and vowel perception. Memory \& Cognition, 3, 7-18.

Postman, L. (1946). The time-error in auditory perception. American Journal of Psychology, 56, 193-219.

Price-Williams, D. R. (1954). The kappa-effect. Nature, 173, 363-364.

REPP, B. H. (1978). Perceptual integration and differentiation of spectral cues for intervocalic stop consonants. Perception \& Psychophysics, 24, 471-485.

Repp, B. H., Healy, A. F., \& Crowder, R. G. (1979). Categories and contexts in the perception of isolated steady-state vowels. Journal of Experimental Psychology: Human Perception \& Performance, $5,129-145$.
Sawusch, J. R., \& NuSBaum, H. C. (1979). Contextual effects in vowel perception I: Anchor-induced contrast effects. Perception \& Psychophysics, 25, 292-302.

Sawusch, J. R., Nusbaum, H. C., \& Schwab, E. C. (1980). Contextual effects in vowel perception II: Evidence for two processing mechanisms. Perception \& Psychophysics, 27, 421-434.

ShIGENo, S., \&UJISAKI, H. (1979). Effect of a preceding anchor upon the categorical judgment of speech and nonspeech stimuli. Japanese Psychological Research, 21, 165-173.

SHIGENo, S., FuJISAKI, H. (1980). A comparison of forward versus backward context effect in phonetic and non-phonetic judgments on synthetic vowels. Japanese Journal of Psychology, 51, 287-290. (In Japanese with English summary)

SimON, H. J., \& STUDDERT-KenNedy, M. (1978). Selective anchoring and adaptation of phonetic and nonphonetic continua. Journal of the Acoustical Society of America, 64, 1338-1357.

(Manuscript received July 22, 1985; revision accepted for publication May 10, 1986.) 